\title{
Intermittent cold stress enhances features of atherosclerotic plaque instability in apolipoprotein E-deficient mice
}

\author{
XI ZHENG* ${ }^{*}$, QIANG WANG* , YAN ZHANG, DACHUN YANG, DE LI, BING TANG, \\ XIUCHUAN LI, YONGJIAN YANG and SHUANGTAO MA
}

Department of Cardiology, General Hospital of PLA Chengdu Military Area Command, Chengdu, Sichuan 610083, P.R. China

Received November 4, 2013; Accepted April 8, 2014

DOI: $10.3892 / \mathrm{mmr} .2014 .2464$

\begin{abstract}
The cold weather is associated with an increased occurrence of acute coronary events. However, the mechanisms underlying cold-induced myocardial infarctions have not yet been fully elucidated. In the present study, 20 male, eight week-old, apolipoprotein E (ApoE)-deficient mice were subjected to either control conditions or intermittent cold exposure for eight weeks. Mice in the cold group were placed in a cold room at $4^{\circ} \mathrm{C}$ for $4 \mathrm{~h}$ per day, while the mice in the control group were kept in a room at $24^{\circ} \mathrm{C}$. Cold-exposed mice did not significantly differ from control mice in body weight, fasting glucose concentration and plasma lipid levels, including triglyceride, total cholesterol, low-density lipoprotein and high-density lipoprotein. The hematoxylin and eosin-stained sections of the aortic root demonstrated increased plaque size in the cold group compared with the control group $(\mathrm{P}<0.01)$. Furthermore, cold-treated mice exhibited significantly decreased plaque collagen and vascular smooth muscle cell deposition and increased macrophage and lymphocyte content $(\mathrm{P}<0.05$ or $\mathrm{P}<0.01$, which are typical features of atherosclerotic plaque instability. Additionally, the protein expression of matrix metalloproteinase (MMP)-2, MMP-9 and MMP-14 were significantly increased $(\mathrm{P}<0.05$ or $\mathrm{P}<0.01)$, whereas tissue inhibitor of matrix metalloproteinase (TIMP)-1 expression was decreased $(\mathrm{P}<0.05)$ following exposure to a cold environment. The present study demonstrated that chronic intermittent cold stress may increase atherosclerotic plaque size and promote plaque instability in ApoE-deficient mice by altering the balance of MMPs and TIMPs. These findings may provide mechanistic insights into sudden cardiac death in cold environments.
\end{abstract}

Correspondence to: Dr Yongjian Yang or Dr Shuangtao Ma, Department of Cardiology, General Hospital of PLA Chengdu Military Area Command, 270 Tianhui Road, Rongdu Avenue, Jinniu, Chengdu, Sichuan 610083, P.R. China

E-mail: yangyongjian38@yahoo.com

E-mail: shuangtaoma@yahoo.com

*Contributed equally

Key words: cold, atherosclerosis, plaque instability, matrix metalloproteinase

\section{Introduction}

Acute coronary syndrome is the leading cause of mortality, not only in developed countries, but also in developing countries, including China (1). Observational epidemiological studies have reported an increased mortality rate from coronary heart disease during winter $(2,3)$. Previous studies have demonstrated that myocardial infarctions increase linearly with a decrease in diurnal temperature $(4,5)$. In addition, a recent study indicated that an extremely cold temperature increases the risk of mortality from ischemic heart disease in China (6).

Although an association between cold temperatures and coronary mortality has been observed, the mechanisms underlying cold-induced myocardial infarctions remain to be fully elucidated. Atherosclerotic plaque rupture is most commonly associated with acute coronary events. The coronary atherosclerotic plaque that is prone to rupture, termed a vulnerable plaque, is a thin-cap fibroatheroma, characterized by a large necrotic core covered by a thin fibrous cap (7). Plaques vulnerable to rupture contain less structural collagen, which is induced by increased production of proteases. Matrix metalloproteinases (MMPs) are the major proteases responsible for weakening the plaque cap. Additionally, MMPs are negatively regulated by intrinsic tissue inhibitor of matrix metalloproteinases (TIMPs). It has been reported that MMPs are upregulated and activated by exposure to a cold environment in several tissues (8). Furthermore, TIMPs appeared to be potent agents in the treatment of cold-induced injury in multiple tissues, including the brain and liver (9-12). Thus, it was hypothesized that cold stress was able to promote atherosclerotic plaque instability associated with an imbalance between MMPs and TIMPs.

In the present study, apolipoprotein E (ApoE)-deficient $\left(\mathrm{ApoE}^{--}\right)$mice were fed a normal diet and exposed to control conditions or a cold environment for eight weeks. The lipid profiles, atherosclerotic plaque size and features of plaque stability were measured at the end of the experiment.

\section{Materials and methods}

Animal care. In total, 20 male, 8-week-old, $\mathrm{ApoE}^{-/-}$mice with a C57BL/6J background, were purchased from Beijing Yikelihao Biotechnology Co., Ltd (Beijing, China). The animals were housed under a $12 \mathrm{~h} / 12 \mathrm{~h}$ day/night cycle with ad libitum access to food and water. The mice were fed a normal chow diet. 
Experimental procedures were approved by the Animal Care and Use Committee of the General Hospital of PLA Chengdu Military Area Command.

Intermittent cold stress. Mice were randomly subjected to room temperature (control group, $\mathrm{n}=10$ ) and intermittent cold stress (cold group, $\mathrm{n}=10$ ) . Mice in the control group were kept in a room with an ambient temperature of $24 \pm 2^{\circ} \mathrm{C}$. Mice in the cold group were placed in a cold room at $4 \pm 1^{\circ} \mathrm{C}$ for $4 \mathrm{~h}$ (between 8:00 am and 10:00 am) and then placed in a room at $24 \pm 2^{\circ} \mathrm{C}$ for the remaining $20 \mathrm{~h}$ per day. The administration period lasted 8 weeks.

Plasma glucose and lipids. Fasting blood samples were obtained at the end of the experiments. Glucose, triglycerides (TG), total cholesterol (TC), low-density lipoprotein-cholesterol (LDL-C) and high-density lipoprotein-cholesterol (HDL-C) were measured by colorimetric assays using commercially available kits (Nanjing Jiancheng Bioengineering Institute, Nanjing, Jiangsu, China) in accordance with the manufacturer's instructions (13).

Atherosclerotic plaque size. The hearts with the aortic roots were harvested and fixed in $10 \%$ paraformaldehyde overnight and embedded in paraffin. Atherosclerotic lesions in the aortic roots were examined in cross-sections of the aortic origin. Six consecutive 5- $\mu \mathrm{m}$-thick sections were cut from the aorta where the valve cusp was visible. The sections were stained with hematoxylin and eosin and images were captured using an Olympus BX41 microscope (Olympus Corp., Tokyo, Japan). The average plaque area was used for statistical analysis (14).

Collagen analysis. Paraffin-embedded aortic sinus sections were stained with Masson's trichrome using a fast Masson dye kit (Nanjing Jiancheng Bioengineering Institute) according to the manufacturer's instructions (15). The percentage of the positively stained area was calculated using Nikon NIS-Elements Research Software (Nikon Instruments, Inc., Melville,. NY, USA).

Immunohistochemistry. Thoracic aortas were fixed in 4\% paraformaldehyde for $12 \mathrm{~h}$, embedded in paraffin and then cut into sections $(5 \mu \mathrm{m})$. The sections were incubated with anti- $\alpha$-smooth muscle-actin ( $\alpha$-SMA; diluted 1:1,500; Epitomics, San Diego, CA, USA), anti-macrophages/monocytes antibody (MOMA)-2 (diluted 1:50; Abcam, Cambridge, MA, USA), anti-cluster of differentiation 3 (CD3; diluted 1:100; GeneTex Inc., San Antonio, TX, USA) antibodies overnight at $4^{\circ} \mathrm{C}$. Specific binding was detected with biotinylated goat anti-rabbit IgG secondary antibody-horseradish peroxidase complexes using an ABC kit (Wuhan Boster Biological Technology, Ltd., Wuhan, Hubei, China). The antigen-antibody complex was subsequently visualized with 3',3'-diaminobenzidine solution. The sections were viewed under a light microscope (Olympus BX41; Olympus Corp., Tokyo, Japan) (16). The percentage of the positively stained area was calculated using Nikon NIS-Elements Research Software.

Western blot analysis. The proteins from the thoracic aorta were extracted using a protein extraction kit (Nanjing KeyGen
Biotech, Co., Ltd., Nanjing, Jiangsu, China). The protein concentrations were determined using an enhanced BCA Protein Assay kit (Beyotime Institute of Biotechnology, Shanghai, China). Extracted protein $(30 \mu \mathrm{g})$ was loaded onto $10 \%$ sodium dodecyl sulfate polyacrylamide gels. The electrophoresis time was decided by the molecular weight of the protein. The separated proteins were then transferred onto appropriate nitrocellulose membranes. The membranes were blocked with Tris-buffered saline with Tween-20 (TBS-T) containing 5\% powdered milk for $1 \mathrm{~h}$ and then incubated with anti-MMP-2, anti-MMP-3, anti-MMP-9, anti-MMP-14, anti-TIMP-1 and anti-TIMP-2 antibodies (Wuhan Boster Biological Technology, Ltd.) at a dilution of 1:200 in 5\% milk/TBS-T overnight. The membranes were rinsed three times with TBS-T and incubated with horseradish peroxidase-conjugated goat antimouse antibody (diluted 1:1,000; Beyotime Institute of Biotechnology) for $1 \mathrm{~h}$. The membranes were rinsed three times with TBS-T and then rinsed once with TBS. Chemiluminescence detection reagent (Millipore, Billerica, MA, USA) was added dropwise onto the membranes. The luminescent signal was detected by exposure to X-ray film (17).

Statistical analysis. Continuous data are presented as the mean \pm standard error of the mean. Comparisons between the two groups were determined by the independent samples t-test (SPSS, Inc., Chicago, IL, USA). P $<0.05$ was considered to indicate a statistically significant difference.

\section{Results}

Metabolic profiles. Whether cold stress affects glycolipid metabolism was initially investigated. At the end of the experiment, mice exposed to a cold environment had a similar body weight compared with the controls (Fig. 1A). Exposure to a cold environment did not affect the fasting plasma glucose concentration (Fig. 1B) and plasma lipid profiles, including TG, TC, LDL-C and HDL-C (Fig. 1C-F; all P>0.05). These findings suggest that cold stress may not affect the glycolipid metabolism of $\mathrm{ApoE}^{-/-}$mice.

Atherosclerotic plaque size. Secondly, the present study investigated whether cold stress affected the growth of atherosclerotic plaques. The atherosclerotic plaque size in the aortic root from the cold group was significantly greater than the control group $(\mathrm{P}<0.01$; Fig. 2). This finding suggested that cold stress may promote plaque growth.

Plaque instability. Thirdly, the present study examined whether cold stress affected the features of atherosclerotic plaque instability. The contents of collagen and vascular smooth muscle cells (SMCs) in atherosclerotic plaques were significantly decreased in the cold group compared with the control group $(\mathrm{P}<0.01$; Fig. 3A, C, E and G), while the contents of macrophages and lymphocytes were significantly increased following 8-week intermittent cold stress $(\mathrm{P}<0.05$ or $\mathrm{P}<0.01$; Fig. 3B, D, F and $\mathrm{H})$. These findings suggest that cold stress may enhance plaque instability.

Expression of MMPS/TIMPs. Finally, it was revealed which molecules may be involved in cold-induced plaque instability. 
A

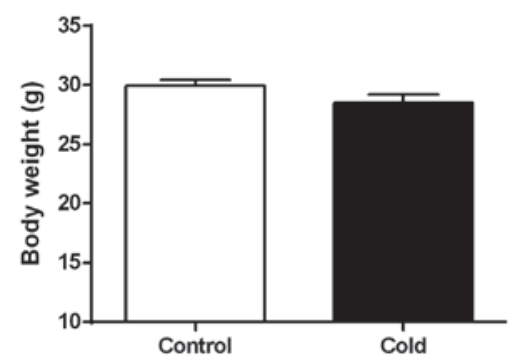

C

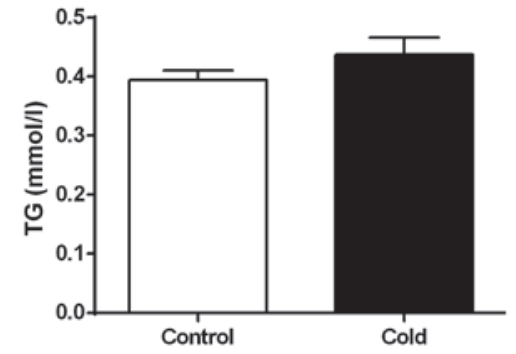

$\mathbf{E}$

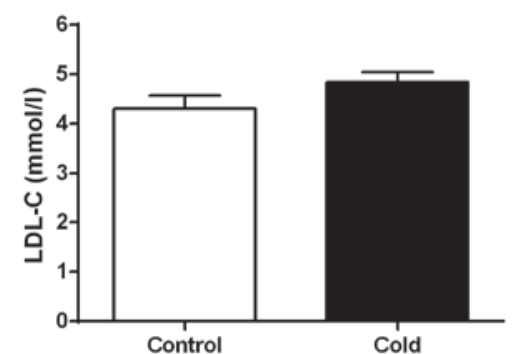

B

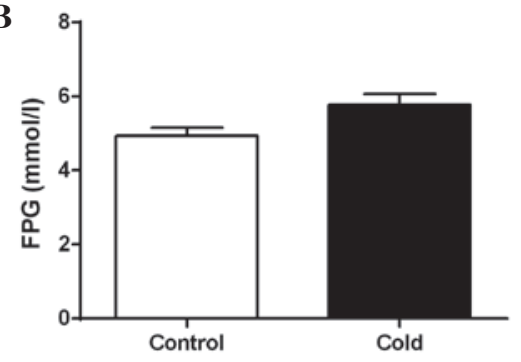

D

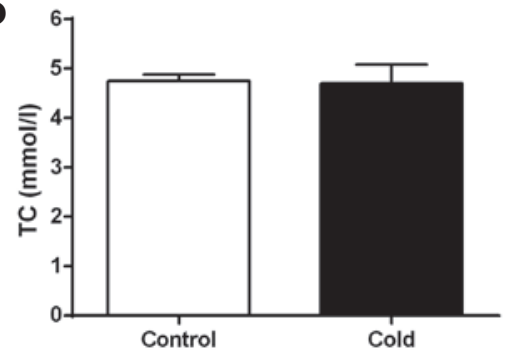

$\mathbf{F}$

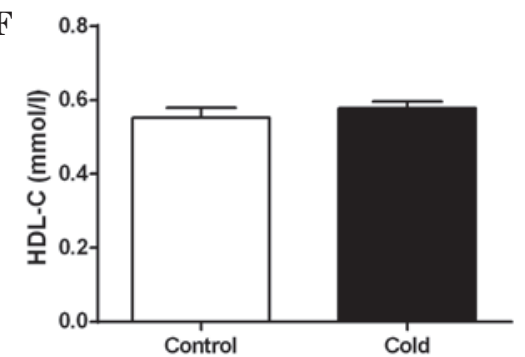

Figure 1. Effects of intermittent cold stress on the metabolic character of ApoE ${ }^{-/}$mice. (A) Body weight, (B) FPG level, (C) TG, (D) TC, (E) LDL-C and (F) HDL-C of ApoE ${ }^{-/}$mice from the control (open bars) and cold (solid bars) groups following 8 weeks of treatment. Data are expressed as the mean \pm standard error of the mean. $n=6$ mice per group. FPG, fasting plasma glucose; LDL-C, low-density lipoprotein cholesterol; HDL-C, high-density lipoprotein cholesterol; TG, triglyceride; TC, total cholesterol.

A

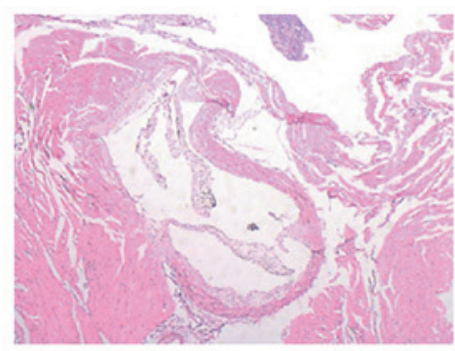

Control

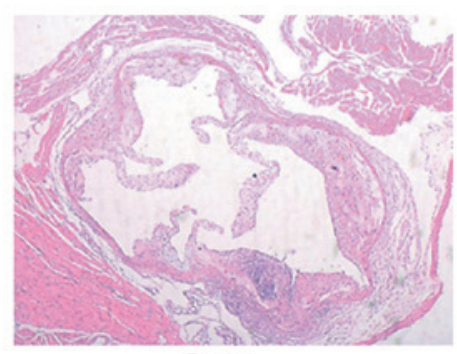

Cold
B

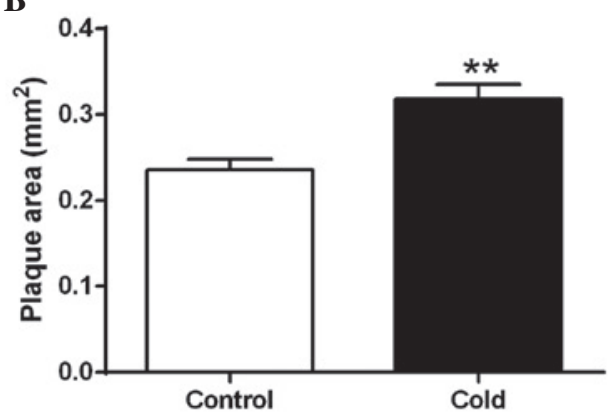

Figure 2. Effects of intermittent cold stress on atherosclerotic plaque size in $\mathrm{ApoE}^{-/}$mice. (A) Representative images of aortic root lesions stained with hematoxylin and eosin (magnification, $x 10$ ). (B) Plaque size of the aortic root is expressed as $\mathrm{mm}^{2}$. Data are expressed as the mean \pm standard error of the mean. $\mathrm{n}=6$ mice per group. ${ }^{* *} \mathrm{P}<0.01$ compared with the control group.

The protein expression of MMP-2, MMP-3, MMP-9, MMP-14, TIMP-1 and TIMP-2 in the thoracic aorta was measured by western blot analysis. Exposure to a cold environment significantly increased the expression of MMP-2, MMP-9 and MMP-14 $(\mathrm{P}<0.01$ or $\mathrm{P}<0.05)$, decreased the expression of TIMP-1 $(\mathrm{P}<0.05)$ and had no significant effect on MMP-3 and TIMP-2 in aortic tissues (Fig. 4). These findings suggest that the imbalance between the protein expression of MMPs and TIMPs in vessels may contribute to cold-induced plaque instability.

\section{Discussion}

The present study demonstrated that chronic intermittent cold stress promotes the growth of atherosclerotic plaques and enhances the features of plaque instability. Additionally, these 
A

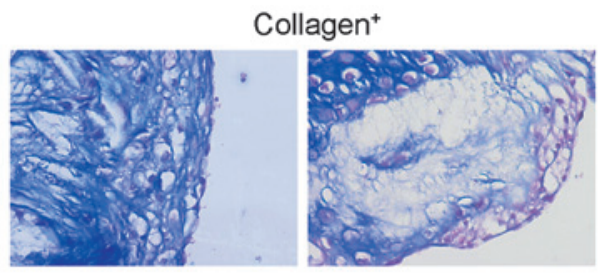

$\mathbf{C}$

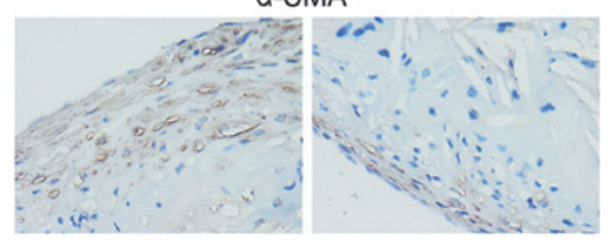

$\mathbf{E}$

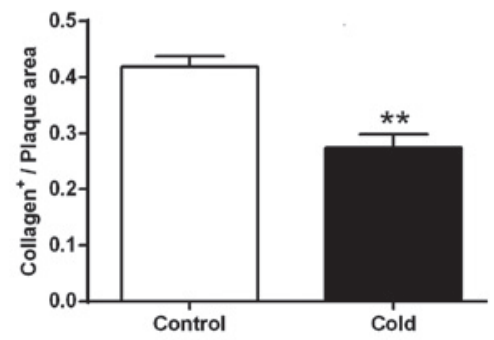

G

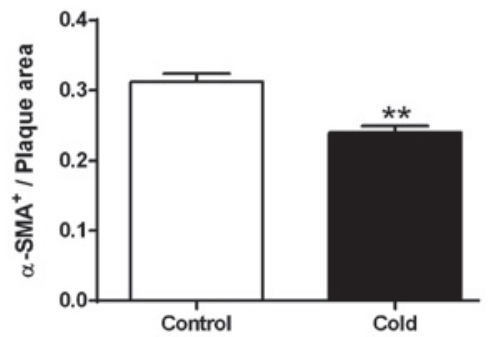

B

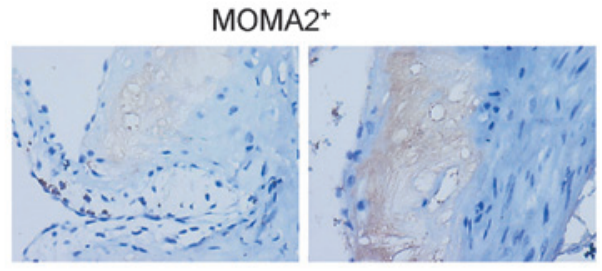

D

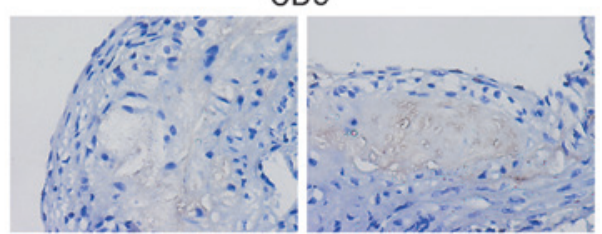

$\mathbf{F}$

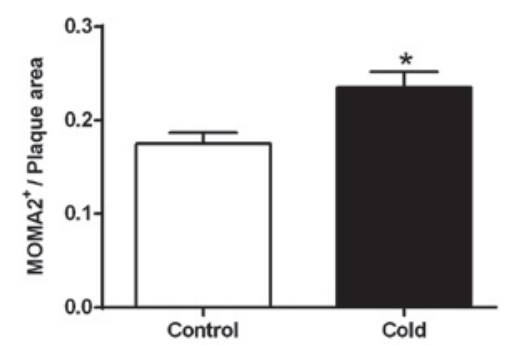

H

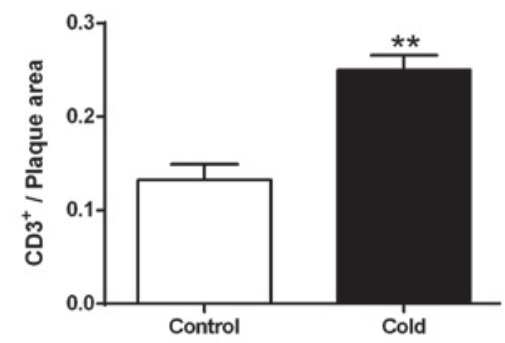

Figure 3. Effects of intermittent cold stress on plaque instability in $\mathrm{ApoE}^{-/}$mice. Representative images of aortic lesions stained with (A) Masson's trichrome, (B) MOMA-2, (C) $\alpha$-SMA or (D) CD3. (E) Collagen, (F) macrophage, (G) vascular smooth muscle cells and (H) lymphocyte depositions were expressed as the percentage of the positively stained area. Data are expressed as the mean \pm standard error of the mean. $\mathrm{n}=6$ mice per group. ${ }^{*} \mathrm{P}<0.05,{ }^{* *} \mathrm{P}<0.01$ compared with the control group. $\alpha$-SMA, $\alpha$-smooth muscle-actin; MOMA-2, macrophages/monocytes antibody-2; CD3, cluster of differentiation 3.

A

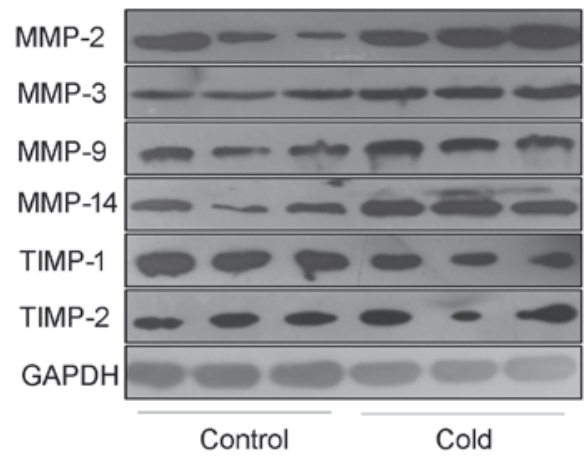

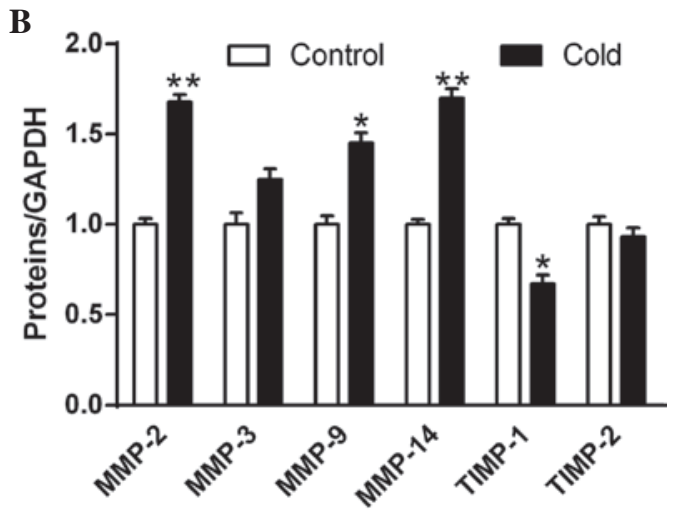

Figure 4. Effects of intermittent cold stress on MMP-2/-3/-9/-14 and TIMP-1/-2 expression in aortas from ApoE ${ }^{-/}$mice. (A) Representative western blots showing the expression of MMP-2, MMP-3, MMP-9, MMP-14, TIMP-1 and TIMP-2 in the aorta. (B) The ratios of proteins/GAPDH are expressed in the bar graph. Data are expressed as the mean \pm standard error of the mean. $\mathrm{n}=5$ mice per group. ${ }^{*} \mathrm{P}<0.05,{ }^{* *} \mathrm{P}<0.01$ compared with the control group. MMP, matrix metalloproteinase; TIMP, tissue inhibitor of metalloproteinase.

effects are associated with the imbalance between MMPs and TIMPs, however, are not associated with obesity, hyperglycemia or hyperlipidemia. These findings suggest that chronic intermittent cold stress may promote plaque instability through enhancing the action of MMPs and subsequently weakening the fibrous cap. 
There is a growing body of evidence that exposure to a cold environment is associated with a high risk of sudden cardiac death. However, a causal link between a cold environment and sudden cardiac death has not been established. The main finding of the present study was that eight-week cold exposure resulted in plaque instability, as indicated by decreased collagen and vascular SMCs deposition and increased macrophage and lymphocyte contents. The fibromuscular cap is a crucial factor for the stability of plaques. Collagen and the vascular SMCs are important parts of the fibrous cap, which are able to resist the mechanical strength and promote the stability of atherosclerotic plaques. These results indicated that a cold environment may induce acute coronary events and sudden cardiac death by increasing the vulnerability of the atherosclerotic plaque.

Metabolic disorders, including obesity, hyperglycemia and hyperlipidemia may promote plaque growth and instability. Chronic cold stress induces adaptive metabolic alterations in several different types of animals. Kozyreva et al (18) reported that cold stress caused a marked decrease in plasma LDL and a significant increase in HDL in hypertensive and normotensive rats. However, a recent study demonstrated that cold stress significantly increased the plasma total cholesterol and LDL in $\mathrm{ApoE}^{-/}$and LDL receptor ${ }^{-/}$mice following persistent exposure to $4^{\circ} \mathrm{C}$ for eight weeks (19). These varied results may be attributed to the different temperatures, exposure time, cooling speed or the genetic background of the animals. In the present study, no effect on body weight, plasma glucose and lipids was observed following eight-week intermittent cold stress. These findings indicated that cold stress-induced plaque growth and instability are not associated with the alterations in glycolipid metabolism.

Hypertension is an important factor in the development of atherosclerosis and the rupture of plaques. Previous studies have demonstrated that chronic cold stress significantly increased systolic and diastolic blood pressure $(20,21)$. However, the present study demonstrated that the blood pressure was similar between the two groups (data not shown). Thus, it was hypothesized that cold-induced plaque instability is not attributed to the alteration in blood pressure.

Mechanisms by which a cold environment causes plaque instability have not been fully elucidated. A previous study demonstrated that chronic cold exposure promotes plaque instability via uncoupling protein 1-dependent lipolysis and hypercholesterolemia (19). However, similar biochemical features were not observed in the present study. Several studies have suggested that MMPs and TIMPs are involved in plaque instability (22-27). Furthermore, MMPs have become recognized as crucial regulators in cold-induced biological processes $(11,12)$. The present study revealed that cold-induced plaque instability was concomitant with the increased expression of MMP-2, MMP-9 and MMP-14 and the decreased expression of TIMP-1. The results from the present study indicated that the imbalance of MMPs and TIMPs may be responsible for the association between intermittent cold stress and plaque vulnerability. Therefore, restoring the balance between MMPs and TIMPs may be a potent method to prevent cold-induced plaque rupture.

In conclusion, the present study demonstrated that chronic intermittent cold exposure increases atherosclerotic plaque size and promotes plaque instability in $\mathrm{ApoE}^{-/-}$mice. Furthermore, the cold stress-induced vulnerability to plaques is associated with the imbalance between MMP-2/-9/-14 and TIMP-1. The present findings may contribute to mechanistic insights into sudden cardiac death in cold environments.

\section{Acknowledgements}

This study was supported by grants from the National Natural Science Foundation of China (nos. 81070191 and 81100232).

\section{References}

1. Bi Y, Gao R, Patel A, et al: Evidence-based medication use among Chinese patients with acute coronary syndromes at the time of hospital discharge and 1 year after hospitalization: results from the Clinical Pathways for Acute Coronary Syndromes in China (CPACS) study. Am Heart J 157: 509-516, 2009.

2. Bhaskaran K, Hajat S, Haines A, Herrett E, Wilkinson P and Smeeth L: Effects of ambient temperature on the incidence of myocardial infarction. Heart 95: 1760-1769, 2009.

3. Barnett AG, Dobson AJ, McElduff P, et al: Cold periods and coronary events: an analysis of populations worldwide. J Epidemiol Community Health 59: 551-557, 2005.

4. De Lorenzo F, Sharma V, Scully M and Kakkar VV: Cold adaptation and the seasonal distribution of acute myocardial infarction. QJM 92: 747-751, 1999.

5. Dilaveris P, Synetos A, Giannopoulos G, Gialafos E, Pantazis A and Stefanadis C: Climate impacts on myocardial infarction deaths in the Athens territory: the CLIMATE study. Heart 92: 1747-1751, 2006.

6. Guo Y, Li S, Zhang Y, et al: Extremely cold and hot temperatures increase the risk of ischaemic heart disease mortality: epidemiological evidence from China. Heart 99: 195-203, 2013.

7. Arbab-Zadeh A, Nakano M, Virmani R and Fuster V: Acute coronary events. Circulation 125: 1147-1156, 2012.

8. Li F, Gao Z, Xu D, Jing J and Wang J: Effect of cold and dry environment on the expression of matrix metalloproteinase 1 mRNA in bone tissues of different pathogenic factors induced models. Zhongguo Zuzhi Gongcheng Yanjiu 16: 9414-9418, 2012.

9. Kawai N, Kawanishi M, Okada M, Matsumoto Y and Nagao S: Treatment of cold injury-induced brain edema with a nonspecific matrix metalloproteinase inhibitor MMI270 in rats. J Neurotrauma 20: 649-657, 2003.

10. Hori T, Uemoto S, Hata T, et al: Matrix metalloproteinase-9 after the cold ischemia/reperfusion injury and/or shear stress with portal hypertension: an overview. Surg Today 44: 201-203, 2013.

11. Upadhya AG, Harvey RP, Howard TK, Lowell JA, Shenoy S and Strasberg SM: Evidence of a role for matrix metalloproteinases in cold preservation injury of the liver in humans and in the rat. Hepatology 26: 922-928, 1997.

12. Li C, Li X, Shen Q, et al: Critical role of matrix metalloproteinase- 9 in acute cold exposure-induced stroke in renovascular hypertensive rats. J Stroke Cerebrovasc Dis 28: e477-e485, 2013.

13. Jiang $\mathrm{S}$, Jin $\mathrm{F}, \mathrm{Li} \mathrm{D}$, et al: Intermittent hypobaric hypoxia promotes atherosclerotic plaque instability in ApoE-deficient mice. High Alt Med Biol 14: 175-180, 2013.

14. Ma S, Yang D, Li D, Tang B and Yang Y: Oleic acid induces smooth muscle foam cell formation and enhances atherosclerotic lesion development via CD36. Lipids Health Dis 10: 53, 2011.

15. Jin F, Jiang S, Yang D, et al: Acipimox attenuates atherosclerosis and enhances plaque stability in ApoE-deficient mice fed a palmitate-rich diet. Biochem Biophys Res Commun 428: 86-92, 2012.

16. Li D, Ma S, Yang Y, et al: BTEB2 knockdown suppresses neointimal hyperplasia in a rat artery balloon injury model. Mol Med Rep 4: 413-417, 2011.

17. Ma S, Yang D, Li D, Tang B, Sun M and Yang Y: Cardiac extracellular matrix tenascin-C deposition during fibronectin degradation. Biochem Biophys Res Commun 409: 321-327, 2011.

18. Kozyreva TV, Lomakina SV, Tuzikov FV and Tuzikova NA: Plasma lipoproteins under the effect of cold exposure in normotensive and hypertensive rats. J Therm Biol 29: 67-72, 2004.

19. Dong M, Yang X, Lim S, et al: Cold exposure promotes atherosclerotic plaque growth and instability via UCP1-dependent lipolysis. Cell Metab 18: 118-129, 2013. 
20. Hintsala H, Kandelberg A, Herzig KH, et al: Central aortic blood pressure of hypertensive men during short-term cold exposure. Am J Hypertens: Aug 20, 2013 (Epub ahead of print).

21. Modesti PA: Season, temperature and blood pressure: A complex interaction. Eur J Intern Med 24: 604-607, 2013.

22. Sapienza P, di Marzo L, Borrelli V, et al: Metalloproteinases and their inhibitors are markers of plaque instability. Surgery 137: 355-363, 2005.

23. Shah PK and Galis ZS: Matrix metalloproteinase hypothesis of plaque rupture: players keep piling up but questions remain. Circulation 104: 1878-1880, 2001.
24. Johnson JL, Fritsche-Danielson R, Behrendt M, et al: Effect of broad-spectrum matrix metalloproteinase inhibition on atherosclerotic plaque stability. Cardiovasc Res 71: 586-595, 2006.

25. Xie S, Nie R and Wang J: Inhibiting extracellular matrix metalloproteinase inducer maybe beneficial for diminishing the atherosclerotic plaque instability. J Postgrad Med 55: 284-286, 2009.

26. Smeglin A and Frishman WH: Elastinolytic matrix metalloproteinases and their inhibitors as therapeutic targets in atherosclerotic plaque instability. Cardiol Rev 12: 141-150, 2004.

27. Loftus IM, Naylor AR, Bell PR and Thompson MM: Matrix metalloproteinases and atherosclerotic plaque instability. Br J Surg 89: 680-694, 2002. 